\title{
New decision-making techniques and their application in the selection of financial products
}

\author{
José M. Merigó*, Anna M. Gil-Lafuente \\ Department of Business Administration, University of Barcelona, Av. Diagonal 690, 08034 Barcelona, Spain
}

\section{A R T I C L E I N F O}

\section{Article history:}

Received 14 August 2008

Received in revised form 26 January 2010

Accepted 27 January 2010

\section{Keywords:}

Decision-making

OWA operator

Selection of financial products

Hamming distance

\begin{abstract}
A B S T R A C T
We develop a new approach that uses the ordered weighted averaging (OWA) operator in the selection of financial products. In doing so, we introduce the ordered weighted averaging distance (OWAD) operator and the ordered weighted averaging adequacy coefficient (OWAAC) operator. These aggregation operators are very useful for decision-making problems because they establish a comparison between an ideal alternative and available options in order to find the optimal choice. The objective of this new model is to manipulate the attitudinal character of previous methods based on distance measures, so that the decision maker can select financial products according to his or her degree of optimism, which is also known as the orness measure. The main advantage of using the OWA operator is that we can generate a parameterized family of aggregation operators between the maximum and the minimum. Thus, the analysis developed in the decision process by the decision maker is much more complete, because he or she is able to select the particular case in accordance with his or her interests in the aggregation process. The paper ends with an illustrative example that shows results obtained by using different types of aggregation operators in the selection of financial products.
\end{abstract}

(c) 2010 Elsevier Inc. All rights reserved.

\section{Introduction}

The selection of more adequate financial products by a company represents a fundamental problem for its future development. With the great variety of alternatives that exist in the market, an enterprise must know which financial products best suit its needs. In order to solve this problem, a company must develop a selection process through which to compare different characteristics of products with the company's needs. Among the great variety of studies on decision-making, this study focuses on (1) the models developed in [11,21] regarding the selection of human resources; (2) the models developed in $[12,13,26]$ regarding the selection of financial products; and (3) the models developed in [14] regarding the selection of players in sport management. Note that these methods follow a methodology similar to the TOPSIS approach [6,19,36] because they are based on the use of distance measures such as the Hamming distance. Note also that in the literature, we find many other decision-making methods [4,15-17,32,38,39,41-43,52,56]; for a general overview of other decision-making methods, refer to [10].

One problem with these selection indices is that they are neutral regarding the attitudinal character of the decision maker. Thus, in the course of the selection process, we cannot manipulate the results according to the interests of the decision maker. This problem becomes important in situations in which we wish to underestimate or overestimate decisions in order to be more or less prudent against uncertain factors affecting the future. One common method for aggregating information regarding the decision attitude of the decision maker is the ordered weighted averaging (OWA) operator [47]. Since its

\footnotetext{
* Corresponding author. Tel.: +349340219 62; fax: +349340398 82.

E-mail addresses: jmerigo@ub.edu (J.M. Merigó), amgil@ub.edu (A.M. Gil-Lafuente).
} 
appearance, the OWA operator has been studied by different authors, including $[1-5,7-9,13,15-18,22-25,27-31,33-$ $35,37,40,46-51,53-55,57-59]$.

Our objective in this paper consists of developing new aggregation operators that include the attitudinal character of the decision maker in the decision process. These new indices consist in combining the OWA operator with the Hamming distance and the adequacy coefficient [21]. We present the ordered weighted averaging distance (OWAD) operator and the ordered weighted averaging adequacy coefficient (OWAAC). These two aggregation operators provide a parameterized family of aggregation operators ranging from the minimum to the maximum. We study some of the main properties of these operators as well as well-known special cases, such as the normalized Hamming distance (NHD), the step-OWAD, the olympicOWAD, the S-OWAD and others. We also prove that the OWAD and the OWAAC converge to the same aggregation under certain conditions.

The main advantage of this new approach is that we generate a parameterized family of aggregation operators for each index, and so the information obtained using this methodology in the selection of financial products is much more complete. Thus, the decision maker is able to consider the decision problem more clearly according to his or her interests in the aggregation process. Another advantage of this approach is the possibility of establishing an ideal, though unrealistic, alternative in order to compare it with available options in the decision-making problem. As such, the optimal choice is the alternative closest to the ideal one.

We also present an illustrative example in order to numerically understand this new approach to the selection of financial products. We use different types of OWAD and OWAAC operators. We see that depending on the particular type of aggregation operator used, the results may lead to different decisions. Note that these aggregation operators can be used in a lot of other problems previously studied with the NHD [20], such as the calculation of distances between intuitionistic fuzzy sets $[39,44,45]$ and interval-valued fuzzy sets.

This paper is organized as follows. In Section 2, we briefly describe the OWA operator. Section 3 explains the basic criteria considered in the selection of financial products using fuzzy techniques. In Sections 4 and 5, we develop a process that uses the OWA operator in the selection of financial products. Section 6 presents an illustrative example of our methodology, and in Section 7, we end the paper with the main conclusions.

\section{The OWA operator}

The OWA operator [47] provides a parameterized family of aggregation operators that have been used in many applications. It can be defined as follows.

Definition 1. An OWA operator of dimension $n$ is a mapping OWA: $R^{n} \rightarrow R$ that has an associated weighting vector $W$ of dimension $n$ having the properties:

(1) $w_{j} \in[0,1]$

(2) $\sum_{j=1}^{n} w_{j}=1$,

such that

$$
\operatorname{OWA}\left(a_{1}, a_{2}, \ldots, a_{n}\right)=\sum_{j=1}^{n} w_{j} b_{j}
$$

where $b_{j}$ is the $j$ th largest of $a_{1} \ldots a_{n}$.

A fundamental aspect of this operator is that it reorders arguments based on their values. That is, rather than being associated with a specific argument as in the case of the usual weighted average, weights here are associated with a particular position in the ordering. This reordering introduces nonlinearity into an otherwise linear process.

Based on the reordering step, we can distinguish between the Descending OWA (DOWA) operator and the Ascending OWA (AOWA) operator. The OWA operator is a mean or averaging operator. This is a reflection of the fact that the operator is commutative, monotonic, idempotent and bounded.

- Commutativity: $\mathrm{OWA}\left(a_{1}, a_{2}, \ldots, a_{n}\right)=\mathrm{OWA}\left(e_{1}, e_{2}, \ldots, e_{n}\right)$, where $\left(e_{1}, e_{2}, \ldots, e_{n}\right)$ is any permutation of the arguments $\left(a_{1}, a_{2}, \ldots, a_{n}\right)$.

- Monotonicity: If $a_{i} \geqslant e_{i}$, for all $i$, then, OWA $\left(a_{1}, a_{2}, \ldots, a_{n}\right) \geqslant \operatorname{OWA}\left(e_{1}, e_{2}, \ldots, e_{n}\right)$.

- Idempotency: If $a_{i}=a$, for all $i$, then, OWA $\left(a_{1}, a_{2}, \ldots, a_{n}\right)=a$.

- Boundedness: $\min \left\{a_{i}\right\} \leqslant \operatorname{OWA}\left(a_{1}, \ldots, a_{n}\right) \leqslant \max \left\{a_{i}\right\}$.

Note that the OWA function is the usual $f$ function. The reason for using our notation is that we want to distinguish it from other types of OWA functions discussed in this paper or in the literature. This should allow decision makers and experts to be able to deal with different types of OWA operators in a more efficient way, because they will only need to look at the exact OWA-name of the $f$ function. For further reading on the theory and application of the OWA operator, refer to [1-5,7-9,13,15$18,22-25,27-31,33-35,37,40,46-51,53-55,57-59]$. 


\section{The selection of financial products using the OWA operator}

The OWA operator is used in the selection of financial products, because the decision maker wants to make his or her decision with a certain degree of optimism rather than neutrality. The traditional methods used in the selection of financial products $[8,20]$ are neutral toward the attitude of the decision maker. Introducing the OWA operator in these models can change the neutrality and thus reflect decisions with different degrees of optimism and pessimism. These techniques can be used in many situations. The importance of this method lies in the possibility of underestimating or overestimating potential problems in order to generate results that reflect changes in evaluation. This can be useful in many situations; for example, the decision maker may want to underestimate results in order to take a more safe approach than in normal cases. Obviously, this increase in safety can affect decisions, as decision makers may select a different financial product than would have been chosen with neutral criteria. Note that in this paper, we focus on the selection of financial products, but the same approach is applicable to other decision-making methods, such as product management and strategic decision-making.

To illustrate how the OWA operator underestimates or overestimates the results, we present a brief example.

Example 1. Assume we want to evaluate a product, and we have three characteristics $C=\left\{C_{1}, C_{2}, C_{3}\right\}$ with values $(0.5,0.8$, 0.2 ). We do not know which characteristics are most important. Therefore, we are in a situation of total uncertainty, and we cannot use probabilities or weighted averages. If we use a neutral approach, we can calculate the average of the characteristics in order to obtain a general result. Thus, $(0.5+0.8+0.2) / 3=0.5$. Sometimes, a decision maker wants to evaluate the product in either an optimistic or a pessimistic way. In this case, he or she will use a weighted average that gives more importance to the highest (optimistic) or the lowest (pessimistic) results. In other words, he or she will use the OWA operator. In this example, assume the following weighting vector: $W=(0.6,0.3,0.1)$. We can evaluate the product in an optimistic way; $0.6 \times 0.8+0.3 \times 0.5+0.1 \times 0.2=0.65$. Or, we can evaluate it in a pessimistic way; $0.6 \times 0.2+0.3 \times 0.5+$ $0.1 \times 0.8=0.35$. Thus, if we evaluate the product in an optimistic way, we generate a higher result $(0.65)$ than if we evaluate it with neutral (0.5) or pessimistic criteria (0.35).

The selection of financial products using the OWA operator is based on the process developed in [11,21] for human resources and in $[12,13,26]$ for financial products, with the difference being that our instruments include the OWA operator in the selection process. Note that we may find in the literature a wide range of other decision-making methods, such as $[4,15-17,32,38,39,41-43,52,56]$. Note also that this approach is presented from the point of view of the selection of financial products, but it may be applied to other decision-making problems, such as strategic decision-making and product management. The five steps of our approach are as follows:

Step 1: Analyze and determine the significant characteristics of available financial products for the company in question. Theoretically, this is represented as: $C=\left\{C_{1}, C_{2}, \ldots, C_{i}, \ldots, C_{n}\right\}$, where $C_{i}$ is the $i$ th characteristic to consider in the financial product. We include a limited number $n$ of required characteristics.

Step 2: Fix the ideal levels of each significant characteristic in order to characterize the ideal financial product. Note that this financial product is not real, but it embodies the best financial product for the decision maker even if it does not exist in the market. This information is presented in Table 1 . where $P$ is the ideal financial product expressed by a fuzzy subset, $C_{i}$ is the $i$ th characteristic under consideration and $\mu_{i} \in[0,1] ; i=1,2, \ldots, n$, is a number between 0 and 1 related to the $i$ th characteristic.

Step 3: Fix the real level of each characteristic for all financial products considered as shown in Table 2. with $k=1,2, \ldots, m$, where $P_{k}$ is the $k$ th financial product expressed by a fuzzy subset, $C_{i}$ is the $i$ th characteristic under consideration, and $\mu_{i}^{(k)} \in[0,1] ; i=1, \ldots, n$, is a number between 0 and 1 related to the $i$ th characteristic of the $k$ th financial product.

Table 1

Ideal financial product.

\begin{tabular}{lllllll}
\hline & $C_{1}$ & $C_{2}$ & $\ldots$ & $C_{i}$ & $\ldots$ & \\
\hline$P=$ & $\mu_{1}$ & $\mu_{2}$ & $\cdots$ & $\mu_{i}$ & $\cdots$ & $\mu_{n}$ \\
\hline
\end{tabular}

Table 2

Available financial products considered.

\begin{tabular}{|c|c|c|c|c|c|c|}
\hline & $C_{1}$ & $C_{2}$ & $\cdots$ & $C_{i}$ & $\ldots$ & $C_{n}$ \\
\hline$P_{1}=$ & $\mu_{1}^{(1)}$ & $\mu_{2}^{(1)}$ & $\cdots$ & $\mu_{i}^{(1)}$ & $\cdots$ & $\mu_{n}^{(1)}$ \\
\hline$\cdots$ & $\cdots$ & $\cdots$ & $\cdots$ & & $\cdots$ & $\cdots$ \\
\hline$P_{k}=$ & $\mu_{1}^{(k)}$ & $\mu_{2}^{(k)}$ & $\cdots$ & $\mu_{i}^{(k)}$ & $\cdots$ & $\mu_{n}^{(k)}$ \\
\hline$\cdots$ & $\cdots$ & $\cdots$ & $\ldots$ & $\ldots$ & $\ldots$ & $\ldots$ \\
\hline$P_{m}=$ & $\mu_{1}^{(m)}$ & $\mu_{2}^{(m)}$ & $\cdots$ & $\mu_{i}^{(m)}$ & $\cdots$ & $\mu_{n}^{(m)}$ \\
\hline
\end{tabular}


Step 4: Compare the ideal financial product and the different financial products under consideration, and determine the distance using the OWA operator. That is, change the neutrality of the results to overestimate or underestimate them. In this step, the objective is to numerically express the difference between the ideal financial product and available financial products under consideration. Different selection indices can be used for this purpose, such as the Hamming distance and the adequacy coefficient. Note that in this paper, we will develop new approaches by using the OWA operator with regard to these indices.

Step 5: Adopt decisions according to the results found in the previous steps. Finally, we must make a decision about which financial product to select. Our decision will involve choosing the financial product with the best results according to the index used.

\section{Using the OWA operator to calculate Hamming distance}

\subsection{Basic concepts}

In this section, we introduce a new index for decision-making using the OWA operator to calculate Hamming distance. We will call it the ordered weighted averaging distance (OWAD) operator. It can be defined as follows for two sets $P$ and $P_{k}$.

Definition 2. An OWAD operator of dimension $n$ is a mapping OWAD : $[0,1]^{n} \times[0,1]^{n} \rightarrow[0,1]$ that has an associated weighting vector $W$, with $\sum_{j=1}^{n} w_{j}=1$ and $w_{j} \in[0,1]$ such that:

$$
\operatorname{OWAD}\left(\left\langle\mu_{1}, \mu_{1}^{(k)}\right\rangle, \ldots,\left\langle\mu_{n}, \mu_{n}^{(k)}\right\rangle\right)=\sum_{j=1}^{n} w_{j} D_{j},
$$

where $D_{j}$ represents the $j$ th largest of the $\left|\mu_{i}-\mu_{i}^{(k)}\right|, \mu_{i} \in[0,1]$ for the $i$ th characteristic of the ideal financial product $P, \mu_{i}^{(k)} \in[0,1]$ for the $i$ th characteristic of the $k$ th financial product considered $P_{k}$, and $k=1,2, \ldots, m$.

Note that this definition can be generalized to all the real numbers $R$ by using OWAD : $R^{n} \times R^{n} \rightarrow R$. Note also that it is possible to distinguish between ascending and descending orders. The weights of these operators are related by $w_{j}=w_{n-j+1}^{*}$, where $w_{j}$ is the $j$ th weight of the descending OWAD (DOWAD) operator and $w_{n-j+1}^{*}$ the $j$ th weight of the ascending OWAD (AOWAD) operator.

Let $B$ be a vector corresponding to the ordered arguments $D_{j}$, we call this the ordered argument vector, and $W^{T}$ is the transpose of the weighting vector. Then the OWAD operator can be expressed as:

$$
\operatorname{OWAD}\left(\left\langle\mu_{1}, \mu_{1}^{(k)}\right\rangle, \ldots,\left\langle\mu_{n}, \mu_{n}^{(k)}\right\rangle\right)=W^{T} B
$$

Note that if the weighting vector is not normalized, i.e., $W=\sum_{j=1}^{n} w_{j} \neq 1$, then, the OWAD operator can be expressed as:

$$
\operatorname{OWAD}\left(\left\langle\mu_{1}, \mu_{1}^{(k)}\right\rangle, \ldots,\left\langle\mu_{n}, \mu_{n}^{(k)}\right\rangle\right)=\frac{1}{W} \sum_{j=1}^{n} w_{j} D_{j} .
$$

Other factors to consider are the measures [47] for characterizing a weighting vector and the type of aggregation it performs. The first measure $\alpha(W)$, the attitudinal character, is defined as:

$$
\alpha(W)=\sum_{j=1}^{n}\left(\frac{n-j}{n-1}\right) w_{j}
$$

It can be shown that $\alpha \in[0,1]$. The more weight is located near the top of $W$, the closer $\alpha$ is to 1 , while the more weight is located toward the bottom of $W$, the closer $\alpha$ is to 0 .

The second measure [47] is called the entropy of dispersion of the weighting vector $W$. It is defined as:

$$
H(W)=-\sum_{j=1}^{n} w_{j} \ln \left(w_{j}\right)
$$

This can be used to measure the quantity of information used. For example, if $w_{j}=1$ for some $j$, then $H(W)=0$, and thus the least amount of information is used.

In addition, the OWAD operator is commutative, monotonic, bounded and idempotent. These properties can be proven with the following theorems:

Theorem 1 (Commutativity). Assume $f$ is the OWAD operator and $\mu_{i}$ and $\mu_{i}^{(k)}$ are the ith values of the sets $P$ and $P_{k}$, then:

$$
f\left(\left\langle\mu_{1}, \mu_{1}^{(k)}\right\rangle, \ldots,\left\langle\mu_{n}, \mu_{n}^{(k)}\right\rangle\right)=f\left(\left\langle\chi_{1}, \chi_{1}^{(k)}\right\rangle, \ldots,\left\langle\chi_{n}, \chi_{n}^{(k)}\right\rangle\right)
$$

where $\left(\left\langle\mu_{1}, \mu_{1}^{(k)}\right\rangle, \ldots,\left\langle\mu_{n}, \mu_{n}^{(k)}\right\rangle\right)$ is any permutation of the arguments $\left(\left\langle\chi_{1}, \chi_{1}^{(k)}\right\rangle, \ldots,\left\langle\chi_{n}, \chi_{n}^{(k)}\right\rangle\right)$.

Proof. It is straightforward and thus omitted. 
Theorem 2 (Monotonicity). Assume $f$ is the OWAD operator and $\mu_{i}$ and $\mu_{i}^{(k)}$ are the ith values of the sets $P$ and $P_{k}$. If $\left|\mu_{i}-\mu_{i}^{(k)}\right| \geqslant\left|\chi_{i}-\chi_{i}^{(k)}\right|$ for all $i$, then:

$$
f\left(\left\langle\mu_{1}, \mu_{1}^{(k)}\right\rangle, \ldots,\left\langle\mu_{n}, \mu_{n}^{(k)}\right\rangle\right) \geqslant f\left(\left\langle\chi_{1}, \chi_{1}^{(k)}\right\rangle, \ldots,\left\langle\chi_{n}, \chi_{n}^{(k)}\right\rangle\right)
$$

Proof. It is straightforward and thus omitted.

Theorem 3 (Idempotency). Assume $f$ is the OWAD operator. If $\left|\mu_{i}-\mu_{i}^{(k)}\right|=d$ for all $i$, then:

$$
f\left(\left\langle\mu_{1}, \mu_{1}^{(k)}\right\rangle, \ldots,\left\langle\mu_{n}, \mu_{n}^{(k)}\right\rangle\right)=d
$$

Proof. It is straightforward and thus omitted.

Theorem 4 (Bounded). Assume $f$ is the OWAD operator, then:

$$
\min \left\{\left|\mu_{i}-\mu_{i}^{(k)}\right|\right\} \leqslant f\left(\left\langle\mu_{1}, \mu_{1}^{(k)}\right\rangle, \ldots,\left\langle\mu_{n}, \mu_{n}^{(k)}\right\rangle\right) \leqslant \max \left\{\left|\mu_{i}-\mu_{i}^{(k)}\right|\right\} .
$$

Proof. Let $\max \left\{\left|\mu_{i}-\mu_{i}^{(k)}\right|\right\}=r$, and $\min \left\{\left|\mu_{i}-\mu_{i}^{(k)}\right|\right\}=s$, then

$$
\begin{aligned}
& f\left(\left\langle\mu_{1}, \mu_{1}^{(k)}\right\rangle, \ldots,\left\langle\mu_{n}, \mu_{n}^{(k)}\right\rangle\right)=\sum_{j=1}^{n} w_{j} D_{j} \leqslant \sum_{j=1}^{n} w_{j} r=r \sum_{j=1}^{n} w_{j}, \\
& f\left(\left\langle\mu_{1}, \mu_{1}^{(k)}\right\rangle, \ldots,\left\langle\mu_{n}, \mu_{n}^{(k)}\right\rangle\right)=\sum_{j=1}^{n} w_{j} D_{j} \geqslant \sum_{j=1}^{n} w_{j} s=s \sum_{j=1}^{n} w_{j} .
\end{aligned}
$$

Since $\sum_{j=1}^{n} w_{j}=1$,

$$
\begin{aligned}
& f\left(\left\langle\mu_{1}, \mu_{1}^{(k)}\right\rangle, \ldots,\left\langle\mu_{n}, \mu_{n}^{(k)}\right\rangle\right) \leqslant r \\
& f\left(\left\langle\mu_{1}, \mu_{1}^{(k)}\right\rangle, \ldots,\left\langle\mu_{n}, \mu_{n}^{(k)}\right\rangle\right) \geqslant s .
\end{aligned}
$$

Therefore,

$$
\min \left\{\left|\mu_{i}-\mu_{i}^{(k)}\right|\right\} \leqslant f\left(\left\langle\mu_{1}, \mu_{1}^{(k)}\right\rangle, \ldots,\left\langle\mu_{n}, \mu_{n}^{(k)}\right\rangle\right) \leqslant \max \left\{\left|\mu_{i}-\mu_{i}^{(k)}\right|\right\} .
$$

\subsection{Families of OWAD operators}

An interesting feature of the OWAD operator is that it provides a parameterized family of distance aggregation operators between the maximum and the minimum. These families use a methodology for establishing the weights similar to the OWA operator. Note that all the methods found in the literature for determining OWA weights are also applicable to the OWAD operator; see [1-4,22-24,27-30,34,35,37,40,48-51,54,55]. Note also that each family is merely a particular case, and as such, they are only useful for specific situations. The main advantage of these families is that we are able to consider particular cases and select which family of operators is most in accordance with our interests in the aggregation process. In the following section, we present some of these families.

Remark 1. By choosing a different manifestation of the weighting vector, we are able to obtain different types of distance aggregation operators. For example, with the DOWAD operator the maximum distance is found when $w_{1}=1$ and $w_{j}=0$ for all $j \neq 1$. The minimum distance is found when $w_{n}=1$ and $w_{j}=0$ for all $j \neq n$.

Remark 2. The step-OWAD sets $w_{k}=1$ and $w_{j}=0$ for all $j \neq k$. Note that if $k=1$, the step-OWAD is transformed to the maximum distance operator, and if $k=n$, the step-OWAD becomes the minimum distance operator.

Remark 3. The normalized Hamming distance is obtained when $w_{j}=1 / n$ for all $j$, and the weighted Hamming distance is obtained when the ordered position of $i$ is the same as the ordered position of $j$. Note that in the case of a tie in the final result, specifically for the maximum and minimum, this operator can be used to decide the second best or worst result.

Remark 4. The olympic-OWAD is generated when $w_{1}=w_{n}=0$, and for all others $w_{j^{*}}=1 /(n-2)$. Following [23], it is possible to develop a general form of the olympic-OWAD by considering that $w_{j}=0$ for $j=1,2, \ldots, k, n, n-1, \ldots, n-k+1$, and for all others $w_{j^{*}}=1 /(n-2 k)$, where $k<n / 2$. Note that if $k=1$, then this general form becomes the usual olympic-OWAD. If $k=(n-1) / 2$, then this general form becomes the median-OWAD aggregation. That is, if $n$ is odd, we assign $w_{(n+1) / 2}=1$, and $w_{j^{*}}=0$ for all other values. If $n$ is even, we assign, for example, $w_{n / 2}=w_{(n / 2)+1}=0.5$ and $w_{j^{*}}=0$ for all other values. 
Remark 5. Following [23], it is also possible to develop the contrary case, that is, the general olympic-OWAD operator. In this case, $w_{j}=(1 / 2 k)$ for $j=1,2, \ldots, k, n, n-1, \ldots, n-k+1$, and $w_{j}=0$, for all other values, where $k<n / 2$. Note that if $k=1$, then we obtain the contrary case for the median-OWAD.

Remark 6. Another interesting family is the S-OWAD operator based on [54]. It can be subdivided into three classes: the "orlike," the "and-like" and the generalized S-OWAD operators. The generalized S-OWAD operator is obtained if $w_{1}=(1 / n)(1-(\alpha+\beta))+\alpha, w_{n}=(1 / n)(1-(\alpha+\beta))+\beta$, and $w_{j}=(1 / n)(1-(\alpha+\beta))$ for $j=2$ to $n-1$, where $\alpha, \beta \in[0,1]$ and $\alpha+\beta \leqslant 1$. Note that if $\alpha=0$, the generalized S-OWAD operator becomes the "and-like" S-OWAD operator, and if $\beta=0$, it becomes the "or-like" S-OWAD operator.

Remark 7. One more interesting family is the non-monotonic-OWAD operator, which follows [49]. It is obtained when at least one of the weights $w_{j}$ is lower than 0 and $\sum_{j=1}^{n} w_{j}=1$. Note that a key aspect of this operator is that it does not always achieve monotonicity. Therefore, strictly speaking, this particular case is not an OWAD operator. However, we can see it as a particular family of operators that is not monotonic but nevertheless resembles an OWAD operator.

\section{Using the OWA operator in the adequacy coefficient}

In this section, we introduce the use of the OWA operator in the selection of financial products using an adequacy coefficient, that is the ordered weighted averaging adequacy coefficient (OWAAC). Note that this operator can be applied to a wide range of problems, such as problems in comparison methods, decision-making problems regarding selection, assignments and groupings [11,12], and business applications in finance and marketing. It can be defined as follows for two sets $P$ and $P_{k}$.

Definition 3. An OWAAC operator of dimension $n$ is a mapping OWAAC: $[0,1]^{n} \times[0,1]^{n} \rightarrow[0,1]$ that has an associated weighting vector $W$, with $w_{j} \in[0,1]$ and $\sum_{j=1}^{n} w_{j}=1$, such that

$$
\operatorname{OWAAC}\left(\left\langle\mu_{1}, \mu_{1}^{(k)}\right\rangle, \ldots,\left\langle\mu_{n}, \mu_{n}^{(k)}\right\rangle\right)=\sum_{j=1}^{n} w_{j} K_{j}
$$

where $K_{j}$ represents the $j$ th largest of $\left[1 \wedge\left(1-\mu_{i}+\mu_{i}^{(k)}\right)\right], \mu_{i} \in[0,1]$, for the $i$ th characteristic of the ideal financial product $P$, $\mu_{i}^{(k)} \in[0,1]$, for the $i$ th characteristic of the $k$ th financial product under consideration and $k=1,2, \ldots, m$.

Note that we can distinguish between descending and ascending orders. The weights of these operators are related by $w_{j}=w_{n-j+1}^{*}$, where $w_{j}$ is the $j$ th weight of the descending OWAAC (DOWAAC) operator, and $w_{n-j+1}^{*}$ is the $j$ th weight of the ascending OWAAC (AOWAAC) operator.

As we can see, the OWAAC operator is commutative, monotonic, bounded and idempotent. Note that it is straightforward to prove these properties by referring to Theorems $1-4$.

By choosing a different configuration of the weighting vector, we are able to obtain different types of aggregation operators. For example, with the DOWAAC operator the maximum is found when $w_{1}=1$ and $w_{j}=0$ for all $j \neq 1$. The minimum is found when $w_{n}=1$ and $w_{j}=0$ for all $j \neq n$. The normalized adequacy coefficient is obtained when $w_{j}=1 / n$ for all $j$. The weighted adequacy coefficient is found when the ordered position of $i$ is the same as the ordered position of $j$.

Other families of OWAAC operators can be obtained using a similar methodology as for the OWAD operator. For example, we could consider the step-OWAAC operator, the window-OWAAC, the olympic-OWAAC, the OWAAC-median, the E-Z OWA$A C$, the centered-OWAAC, the S-OWAAC or the non-monotonic-OWAAC. The formulation of these families is straightforward, as indicated in Remarks 2-7 and in [1-4,22-24,27-30,34,35,37,40,48-51,54,55].

Analogously to the OWAAC operator, we can suggest a removal index that is the dual of the OWAAC operator, because $Q\left(\left\langle\mu_{1}, \mu_{1}^{(k)}\right\rangle, \ldots,\left\langle\mu_{n}, \mu_{n}^{(k)}\right\rangle\right)=1-K\left(\left\langle\mu_{1}, \mu_{1}^{(k)}\right\rangle, \ldots,\left\langle\mu_{n}, \mu_{n}^{(k)}\right\rangle\right)$. We will call it the ordered weighted averaging dual adequacy coefficient (OWADAC). It is defined as follows.

Definition 4. An OWADAC operator of dimension $n$ is a mapping OWADAC: $[0,1]^{n} \times[0,1]^{n} \rightarrow[0,1]$ that has an associated weighting vector $W$, with $w_{j} \in[0,1]$ and $\sum_{j=1}^{n} w_{j}=1$, such that

$$
\operatorname{OWADAC}\left(\left\langle\mu_{1}, \mu_{1}^{(k)}\right\rangle, \ldots,\left\langle\mu_{n}, \mu_{n}^{(k)}\right\rangle\right)=\sum_{j=1}^{n} w_{j} Q_{j},
$$

where $Q_{j}$ represents the $j$ th largest of $\left[0 \vee\left(\mu_{i}-\mu_{i}^{(k)}\right)\right], \mu_{i} \in[0,1]$, for the $i$ th characteristic of the ideal financial product $P$, $\mu_{i}^{(k)} \in[0,1]$, for the $i$ th characteristic of the $k$ th financial product considered, and $k=1,2, \ldots, m$.

In this case, we can also distinguish between the descending OWADAC (DOWADAC) operator and the ascending OWADAC (AOWADAC) operator by using $w_{j}=w_{n-j+1}^{*}$, where $w_{j}$ is the $j$ th weight of the DOWADAC operator and $w_{n-j+1}^{*}$ is the $j$ th weight of the AOWADAC operator.

Note that it is also possible to obtain different families of aggregation operators with the OWADAC operator by using different configurations of the weighting vector, such as the maximum, the minimum, the normalized dual adequacy coefficient (NDAC), or the general olympic-OWADAC. 
Another interesting issue to consider is the unification point between the Hamming distance and the adequacy coefficient in the selection of financial products [26]. As has been explained in [26], the unification point appears when the results obtained in the Hamming distance are the same as the results obtained using the adequacy coefficient. According to the new methods suggested in this paper, we also find the unification point when the OWAD and the OWAAC operators fulfill the conditions of the theorems presented in [26]. Note that it is possible to find a total unification point or a partial unification point: we consider the unification point for one financial product.

Theorem 5. Assume $\operatorname{OWAD}\left(\left\langle\mu_{1}, \mu_{1}^{(k)}\right\rangle, \ldots,\left\langle\mu_{n}, \mu_{n}^{(k)}\right\rangle\right)$ is the selection of financial products with the OWAD operator, and $\operatorname{OWADAC}\left(\left\langle\mu_{1}, \mu_{1}^{(k)}\right\rangle, \ldots,\left\langle\mu_{n}, \mu_{n}^{(k)}\right\rangle\right)$ is the selection of financial products with the OWADAC operator. If $\mu_{i} \geqslant \mu_{i}^{(k)}$ for all $i$, then:

$$
\operatorname{OWAD}\left(\left\langle\mu_{1}, \mu_{1}^{(k)}\right\rangle, \ldots,\left\langle\mu_{n}, \mu_{n}^{(k)}\right\rangle\right)=\operatorname{OWADAC}\left(\left\langle\mu_{1}, \mu_{1}^{(k)}\right\rangle, \ldots,\left\langle\mu_{n}, \mu_{n}^{(k)}\right\rangle\right) .
$$

Proof. Let

$$
\begin{aligned}
& \operatorname{OWAD}\left(\left\langle\mu_{1}, \mu_{1}^{(k)}\right\rangle, \ldots,\left\langle\mu_{n}, \mu_{n}^{(k)}\right\rangle\right)=\sum_{j=1}^{n} w_{j}\left|\mu_{i}-\mu_{i}^{(k)}\right| \text { and } \\
& \operatorname{OWADAC}\left(\left\langle\mu_{1}, \mu_{1}^{(k)}\right\rangle, \ldots,\left\langle\mu_{n}, \mu_{n}^{(k)}\right\rangle\right)=\sum_{j=1}^{n} w_{j}\left[0 \vee\left(\mu_{i}-\mu_{i}^{(k)}\right)\right] .
\end{aligned}
$$

Since $\mu_{i} \geqslant \mu_{i}^{(k)}$ for all $i,\left[0 \vee\left(\mu_{i}-\mu_{i}^{(k)}\right)\right]=\left(\mu_{i}-\mu_{i}^{(k)}\right)$ for all $i$, then

$$
\operatorname{OWADAC}\left(\left\langle\mu_{1}, \mu_{1}^{(k)}\right\rangle, \ldots,\left\langle\mu_{n}, \mu_{n}^{(k)}\right\rangle\right)=\sum_{j=1}^{n} w_{j}\left(\mu_{i}-\mu_{i}^{(k)}\right)=\operatorname{OWAD}\left(\left\langle\mu_{1}, \mu_{1}^{(k)}\right\rangle, \ldots,\left\langle\mu_{n}, \mu_{n}^{(k)}\right\rangle\right) .
$$

Remark 8. We can generalize this theorem for all financial products considered in the decision problem under consideration. The theorem that justifies this generalization is very similar to Theorem 5 , with the difference being that now we consider all characteristics $i$ and all financial products $k$.

\section{Numerical example}

Information about the example presented in this paper is found in [12]; note, however, that we have made some changes for the purposes of this paper.

Step 1: Analyze and determine the significant characteristics regarding the financial products for the company under consideration.It is assumed that in a financial market, three financial products $P_{1}, P_{2}$ and $P_{3}$ exist with different characteristics as follows:

- $C_{1}=$ prize.

- $C_{2}=$ devolution period.

- $C_{3}=$ attributes related to renewal or reactivation.

- $C_{4}=$ the break up of amortization.

- $C_{5}=$ velocity in the concession.

Step 2: Fix the ideal level of each significant characteristic, thereby defining the ideal product for the company using fuzzy subsets as shown in Table 3 .

Step 3: Fix the actual level of each characteristic for all financial products under consideration. For each characteristic, we find the following information:

- For $C_{1}$ : The price of $P_{1}$ is $20 \%$, of $P_{2}$ is $22 \%$ and of $P_{3}$ is $18 \%$.

- For $C_{2}$ : The devolution period of $P_{1}$ is 5 years, of $P_{2}$ is 6 years and of $P_{3}$ is 4 years.

- For $C_{3}$ : The renewal possibilities of $P_{1}$ are half of $P_{2}$ and $1 / 3$ of $P_{3}$.

- For $C_{4}$ : The amortization of $P_{1}$ is conducted quarterly, for $P_{2}$, it is conducted monthly, and for $P_{3}$ it is conducted quarterly.

- For $C_{5}$ : The renewal of $P_{1}$ is estimated to be three times faster than $P_{2}$ and five times faster than $P_{3}$.

With this information, we can obtain a fuzzy subset for each financial products. That is, we will obtain the following results as shown in Table 4.

These results show the level of each product regarding each characteristics considered, that is, $C_{i}$, for $i=1,2,3,4,5$.

Step 4: Compare the ideal financial product and the actual products under consideration, and determine the level of removal using the OWA operator. We consider the normalized Hamming distance, the weighted Hamming distance, the OWAD operator and the AOWAD operator. In this example, we assume that the company decides to use the following weighting vector: $W=(0.1,0.1,0.2,0.3,0.3)$. With this weighting vector, we calculate the degree 
Table 3

Ideal financial product.

\begin{tabular}{lllll}
\hline & $C_{1}$ & $C_{2}$ & $C_{3}$ & $C_{5}$ \\
\hline$P^{*}=$ & 0.9 & 0.83 & 0.66 & 0.66 \\
\hline
\end{tabular}

Table 4

Available financial products.

\begin{tabular}{lllll}
\hline & $C_{1}$ & $C_{2}$ & $C_{3}$ & $C_{4}$ \\
\hline$P_{1}=$ & 0.9 & 0.83 & 0.33 & 1 \\
$P_{2}=$ & 0.81 & 1 & 0.66 & 0.33 \\
$P_{3}=$ & 1 & 0.66 & 1 & 0.33 \\
\hline
\end{tabular}

Table 5

Aggregated results with the Hamming distance.

\begin{tabular}{llll}
\hline & NHD & WHD & OWAD \\
\hline$P_{1}$ & 0.266 & 0.366 & 0.166 \\
$P_{2}$ & 0.118 & 0.126 & 0.068 \\
$P_{3}$ & 0.212 & 0.232 & 0.168 \\
\hline
\end{tabular}

Table 6

Aggregated results with the adequacy coefficient.

\begin{tabular}{llll}
\hline & NAC & WAC & OWAAC \\
\hline$P_{1}$ & 0.933 & 0.933 & 0.898 \\
$P_{2}$ & 0.915 & 0.891 & 0.871 \\
$P_{3}$ & 0.938 & 0.943 & 0.966 \\
\hline
\end{tabular}

Table 7

Aggregated results with the dual adequacy coefficient.

\begin{tabular}{llll}
\hline & NDAC & WDAC & OWADAC \\
\hline$P_{1}$ & 0.067 & 0.067 & 0.102 \\
$P_{2}$ & 0.085 & 0.109 & 0.129 \\
$P_{3}$ & 0.062 & 0.057 & 0.093 \\
\hline
\end{tabular}

of optimism of the decision as $\alpha(W)=0.35=35 \%$, while the degree of dispersion is $H(W)=1.504$.

If we elaborate the selection process using the Hamming distance, we obtain the following. First, we must calculate the individual distances of each characteristic from the ideal value of the corresponding characteristic, thereby forming the fuzzy subset of individual distances for each financial product. Once all distances are obtained, we can aggregate. We reorder the different values of each fuzzy subset using Eq. (2) in line with the type of aggregation we are interested in conducting. The results are shown in Table 5.

In this case, our decision consists in selecting the financial product with the smallest distance. We select $P_{2}$, as it gives us the minimum distance across the four cases.

If we develop the selection process using the adequacy coefficient, we generate the following. First, we must calculate how close the characteristics are to the ideal financial product. Once all individual values are calculated, we aggregate the results. In this case, the arguments are ordered using Eq. (15). The results of this process are shown in Table 6.

The decision consists in selecting the financial product with the highest result, because this indicates a higher approximation to the ideal financial product. Thus, we select $P_{3}$, because it offers the highest result across all cases.

Analogous to this index, we can obtain its equivalent removal index. In an abbreviated form, this index can be obtained by noting that $Q\left(P_{k}, P\right)=1-K\left(P_{k}, P\right)$. These results are shown in Table 7 .

Step 5: Depending on the aggregation operator, the results may lead to different decisions. Thus, by using these decisionmaking techniques based on the OWA operator, we can present a wide range of methods for the decision maker. As such, he or she can consider a wide range of situations and select the one that it is in accordance with his or her interests. 


\section{Conclusions}

We have studied new decision-making techniques, and we have applied them in a decision-making problem regarding the selection of financial products. We have presented the OWAD and OWAAC operators as new types of aggregation operators that are useful in a wide range of applications, such as decision-making. The main advantage of this approach is that we are able to underestimate or overestimate the selection process according to the desired degree of optimism (i.e., the degree of orness). Therefore, we are able to provide decision maker with a method that considers the optimal choice according to his or her interests. Moreover, with this approach, we are able to consider a wide range of scenarios, because the OWAD and OWAAC operators provide a parameterized family of aggregation operators between the minimum and the maximum. Thus, the decision maker is able to obtain a more complete picture of the decision-making problem at hand.

We have studied different properties of these operators, such as the distinction between descending and ascending orders as well as different families of aggregation operators, such as the step-OWAD and the olympic-OWAD. We have seen that under certain conditions, the OWAD and OWAAC operators converge toward the same aggregation operator. We have also presented a numerical example in order to show the applicability of the new approach in the selection of financial products. We have seen that depending on the particular case used, the results may lead to different decisions.

This work represents an initial analysis regarding the possibility of using the OWA operator in different selection indices. In this paper, we have focussed on the selection of financial products, but it is important to note that these new methods can also be applied to other selection processes, such as the selection of human resources, strategies, and investments. In future research, we will analyze how these methods can be applied to other selection processes, and we will consider other selection indices as well as other types of OWA operators.

\section{Acknowledgements}

We would like to thank the Editor-in-Chief, Professor Witold Pedrycz, and the anonymous referees for their valuable comments and suggestions, which led to an improved version of the paper. Financial support from the Spanish Ministerio de Asuntos Exteriores y de Cooperacion, Agencia Española de Cooperación Internacional para el Desarrollo (AECID) (Project A/016239/08), is gratefully acknowledged.

\section{References}

[1] B.S. Ahn, The OWA aggregation with uncertain descriptions on weights and input arguments, IEEE Transactions on Fuzzy Systems 15 (2007) 11301134.

[2] B.S. Ahn, H. Park, Least-squared ordered weighted averaging operator weights, International Journal of Intelligent Systems 23 (2008) $33-49$.

[3] G. Beliakov, A. Pradera, T. Calvo, Aggregation Functions: A Guide for Practitioners, Springer-Verlag, Berlin, 2007.

[4] T. Calvo, G. Mayor, R. Mesiar, Aggregation Operators: New Trends and Applications, Physica-Verlag, New York, 2002.

[5] L. Canós, V. Liern, Soft computing-based aggregation methods for human resource management, European Journal of Operational Research 189 (2008) 669-681.

[6] T.Y. Chen, C.Y. Tsao, The interval-valued fuzzy TOPSIS method and experimental analysis, Fuzzy Sets and Systems 159 (2008) $1410-1428$.

[7] F. Chiclana, F. Herrera, E. Herrera-Viedma, S. Alonso, Induced ordered weighted geometric operators and their use in the aggregation of multiplicative preference relations, International Journal of Intelligent Systems 19 (2004) 233-255.

[8] F. Chiclana, F. Herrera, E. Herrera-Viedma, L. Martínez, A note on the reciprocity in the aggregation of fuzzy preference relations using OWA operators, Fuzzy Sets and Systems 137 (2003) 71-83.

[9] F. Chiclana, E. Herrera-Viedma, F. Herrera, S. Alonso, Some induced ordered weighted averaging operators and their use for solving group decisionmaking problems based on fuzzy preference relations, European Journal of Operational Research 182 (2007) 383-399.

[10] J. Figueira, S. Greco, M. Ehrgott, Multiple Criteria Decision Analysis: State of the Art Surveys, Springer, Boston, 2005.

[11] J. Gil-Aluja, The Interactive Management of Human Resources in Uncertainty, Kluwer Academic Publishers, Dordrecht, 1998.

[12] A.M. Gil-Lafuente, Fuzzy Logic in Financial Analysis, Springer, Berlin, 2005.

[13] A.M. Gil-Lafuente, J.M. Merigó, Acquisition of financial products that adapt to different environments, Lectures on Modelling and Simulation 7 (2006) $42-48$.

[14] J. Gil-Lafuente, The index of maximum and minimum level in the selection of players in sport management (in Spanish), in: Proceedings of 10th International Conference of the European Academy of Management and Business Economics (AEDEM), Reggio Calabria, Italy, 2001, pp. 439-443.

[15] F. Herrera, E. Herrera-Viedma, F. Chiclana, A study of the origin and uses of the ordered weighted geometric operator in multicriteria decision making, International Journal of Intelligent Systems 18 (2003) 689-707.

[16] E. Herrera-Viedma, S. Alonso, F. Chiclana, F. Herrera, A consensus model for group decision making with incomplete fuzzy preference relations, IEEE Transactions on Fuzzy Systems 15 (2007) 863-877.

[17] E. Herrera-Viedma, F. Chiclana, F. Herrera, S. Alonso, Group decision-making model with incomplete fuzzy preference relations based on additive consistency, IEEE Transactions on Systems, Man and Cybernetics B 37 (2007) 176-189.

[18] Y.C. Hu, J.F. Tsai, Fusing fuzzy association rule-based classifiers using Sugeno integrals with ordered weighted averaging operators, International Journal of Uncertainty, Fuzziness and Knowledge-Based Systems 15 (2007) 717-735.

[19] C.L. Hwang, K. Yoon, Multiple Attribute Decision Making: Methods and Applications, Springer-Verlag, Berlin, 1981.

[20] A. Kaufmann, Introduction to the Theory of Fuzzy Subsets, Academic Press, New York, 1975.

[21] A. Kaufmann, J. Gil-Aluja, Introduction to the Theory of Fuzzy Subsets in Business Management (in Spanish), Milladoiro, Santiago de Compostela, 1986.

[22] X. Liu, The solution equivalence of minimax disparity and minimum variance problems for OWA operators, International Journal of Approximate Reasoning 45 (2007) 68-81.

[23] X. Liu, Parameterized OWA operator determination with optimization criteria: a general method, IEEE Transactions on Fuzzy Systems, in press.

[24] X. Liu, S. Han, Orness and parameterized RIM quantifier aggregation with OWA operators: a summary, International Journal of Approximate Reasoning 48 (2008) 77-97.

[25] B. Llamazares, Choosing OWA operator weights in the field of social choice, Information Sciences 177 (2007) 4745-4756.

[26] J.M. Merigó, A.M. Gil-Lafuente, Unification point in methods for the selection of financial products, Fuzzy Economic Review 12 (2007) 35-50. 
[27] J.M. Merigó, A.M. Gil-Lafuente, On the use of the OWA operator in the Euclidean distance, International Journal of Computer Science and Engineering 2 (2008) $170-176$.

[28] J.M. Merigó, A.M. Gil-Lafuente, Using the OWA operator in the Minkowski distance, International Journal of Computer Science 3 (2008) 149-157.

[29] J.M. Merigó, A.M. Gil-Lafuente, The generalized adequacy coefficient and its application in strategic decision making, Fuzzy Economic Review 13 (2008) $17-36$.

[30] J.M. Merigó, A.M. Gil-Lafuente, The induced generalized OWA operator, Information Sciences 179 (2009) 729-741.

[31] H.B. Mitchell, D.D. Estrakh, An OWA operator with fuzzy ranks, International Journal of Intelligent Systems 13 (1998) 69-81.

[32] D. Pelta, R.R. Yager, On the conflict between inducing confusion and attaining payoff in adversarial decision making, Information Sciences 179 (2009) 33-40.

[33] X. Wang, Fuzzy number intuitionistic fuzzy arithmetic aggregation operators, International Journal of Fuzzy Systems 10 (2008) $104-111$.

[34] Y.M. Wang, C. Parkan, A preemptive goal programming method for aggregating OWA operator weights in group decision making, Information Sciences 177 (2007) 1867-1877.

[35] J. Wu, C.Y. Liang, Y.Q. Huang, An argument-dependent approach to determining OWA operator weights based on the rule of maximum entropy, International Journal of Intelligent Systems 22 (2007) 209-221.

[36] W.Y. Wu, C. Lin, J.Y. Kung, C.T. Lin, A new fuzzy TOPSIS for fuzzy MADM problems under group decisions, Journal of Intelligent \& Fuzzy Systems 18 (2007) 109-115.

[37] Z.S. Xu, An overview of methods for determining OWA weights, International Journal of Intelligent Systems 20 (2005) 843-865.

[38] Z.S. Xu, Intuitionistic preference relations and their application in group decision making, Information Sciences 177 (2007) 2363-2379.

[39] Z.S. Xu, Some similarity measures of intuitionistic fuzzy sets and their applications to multiple attribute decision making, Fuzzy Optimization and Decision Making 6 (2007) 109-121.

[40] Z.S. Xu, Dependent uncertain ordered weighted averaging operators, Information Fusion 9 (2008) 310-316

[41] Z.S. Xu, On multi-period multi-attribute decision making, Knowledge-Based Systems 21 (2008) 164-171.

[42] Z.S. Xu, Group decision making based on multiple types of linguistic preference relations, Information Sciences 178 (2008) $452-467$.

[43] Z.S. Xu, J. Chen, An interactive method for fuzzy multiple attribute group decision making, Information Sciences 177 (2007) $248-263$.

[44] Z.S. Xu, J. Chen, An overview of distance and similarity measures of intuitionistic fuzzy sets, International Journal of Uncertainty, Fuzziness and Knowledge-Based Systems 16 (2008) 529-555.

[45] Z.S. Xu, J. Chen, J. Wu, Clustering algorithm for intuitionistic fuzzy sets, Information Sciences 178 (2008) 3775-3790.

[46] Z.S. Xu, Q.L. Da, An overview of operators for aggregating information, International Journal of Intelligent Systems 18 (2003) 953-969.

[47] R.R. Yager, On ordered weighted averaging aggregation operators in multi-criteria decision making, IEEE Transactions on Systems, Man and Cybernetics B 18 (1988) 183-190.

[48] R.R. Yager, Families of OWA operators, Fuzzy Sets and Systems 59 (1993) 125-148.

[49] R.R. Yager, Nonmonotonic OWA operators, Soft Computing 3 (1999) 187-196.

[50] R.R. Yager, Centered OWA operators, Soft Computing 11 (2007) 631-639.

[51] R.R. Yager, Using stress functions to obtain OWA operators, IEEE Transactions on Fuzzy Systems 15 (2007) 1122-1129.

[52] R.R. Yager, A knowledge-based approach to adversarial decision making, International Journal of Intelligent Systems 23 (2008) 1-21.

[53] R.R. Yager, Using trapezoids for representing granular objects: applications to learning and OWA aggregation, Information Sciences 178 (2008) $363-$ 380.

[54] R.R. Yager, D.P. Filev, Parameterized "andlike" and "orlike" OWA operators, International Journal of General Systems 22 (1994) $297-316$.

[55] R.R. Yager, J. Kacprzyk, The Ordered Weighted Averaging Operators: Theory and Applications, Kluwer Academic Publishers, Norwell, MA, 1997.

[56] J.L. Yang, H.N. Chiu, G.H. Tzeng, R.H. Yeh, Vendor selection by integrated fuzzy MCDM techniques with independent and interdependent relationships, Information Sciences 178 (2008) 4166-4183.

[57] D.Y. Yeh, C.H. Cheng, H.W. Yio, Empirical research of the principal component analysis and ordered weighted averaging integrated evaluation model on software projects, Cybernetics and Systems 38 (2007) 289-303.

[58] M. Zarghami, F. Szidarovszky, R. Ardakanian, A fuzzy-stochastic OWA model for robust multi-criteria decision making, Fuzzy Optimization and Decision Making 7 (2008) 1-15.

[59] S.M. Zhou, F. Chiclana, R.I. John, J.M. Garibaldi, Type-1 OWA operators for aggregating uncertain information with uncertain weights induced by type-2 linguistic quantifiers, Fuzzy Sets and Systems 159 (2008) 3281-3296. 